ÉGYPTE monde arabe

\section{Égypte/Monde arabe}

15-16 | 1993

Les crises soudanaises des années 80

\title{
La « tribu » des diplômés et la mauvaise gestion de l'économie soudanaise
}

\section{Siddig Umbadda}

\section{(2) OpenEdition}

\section{Journals}

Édition électronique

URL : https://journals.openedition.org/ema/1097

DOI : 10.4000/ema. 1097

ISSN : 2090-7273

\section{Éditeur}

CEDEJ - Centre d'études et de documentation économiques juridiques et sociales

\section{Édition imprimée}

Date de publication : 31 décembre 1993

Pagination : 191-206

ISSN : 1110-5097

\section{Référence électronique}

Siddig Umbadda, «La «tribu » des diplômés et la mauvaise gestion de l'économie soudanaise »,

Égypte/Monde arabe [En ligne], 15-16 | 1993, mis en ligne le 08 juillet 2008, consulté le 07 juillet 2022. URL : http://journals.openedition.org/ema/1097 ; DOI : https://doi.org/10.4000/ema.1097

Ce document a été généré automatiquement le 7 juillet 2022.

Tous droits réservés 


\title{
La « tribu » des diplômés et la mauvaise gestion de l'économie soudanaise
}

\author{
Siddig Umbadda
}

\section{NOTE DE L'ÉDITEUR}

Traduit de l'anglais par Alain Roussillon.

1 Un coup d'œil, même rapide, aux indicateurs macro-économiques depuis l'indépendance suffit à convaincre que l'économie soudanaise - compte tenu des ressources existantes - n'a pas été bien gérée ${ }^{1}$. Non seulement l'on y constate que le «gâteau national » - le PNB - n'a pas augmenté de façon suffisamment rapide, mais la distribution de ses parts a été injuste. Les inégalités entre les régions et les groupes socio-ethniques dans l'accès au pouvoir et à la richesse ont donc alimenté les insatisfactions et donné naissance à des conflits parfois violents, qui ont encore ralenti la croissance de l'économie.

2 Si les facteurs concourant à ces tristes performances économiques sont nombreux, les facteurs internes peuvent se voir assigner un rôle majeur. La présente étude propose une explication partielle des causes qui permettent de comprendre pourquoi l'économie sous direction nationale n'a pas réussi à décoller. Principale hypothèse : la centralité du rôle de l'élite. Le type d'éducation reçu par les premiers dirigeants soudanais, ainsi que leur appartenance socio-ethnique et régional, sont ici considérés comme l'un des facteurs permettant de rendre compte de l'attitude générale de " quête de rente » qui caractérise leurs positions par rapport aux enjeux nationaux majeurs.

3 En première partie, nous présentons le cadre d'analyse dans lequel nous tentons de rendre compte des performances de l'économie soudanaise après l'indépendance. La deuxième partie examine le degré de concentration du pouvoir d'État dans les mains de 
Soudanais originaires de certaines régions. La troisième partie envisage les implications de la gestion déficiente de l'économie.

Cadre d'analyse

4 Les bénéfices de l'instruction, en général, et de l'instruction supérieure, en particulier, sont généralement envisagés en termes de mobilité sociale et d'accroissement des revenus qui en découlent (accès aux emplois en fonction du mérite). En fait, on a montré que le coût d'une éducation supérieure donnée à des pauvres était plus élevé, et les bénéfices retirés par eux de cette éducation plus faibles (Bhagwati, 1985), dans la mesure où, entre autres raisons, les enfants des groupes à haut revenu sont en situation d'obtenir (à qualification équivalente) les meilleurs emplois et/ou d'être recrutés plus rapidement. On peut s'accorder à qualifier de "rente" ces bénéfices supérieurs obtenus par voie d'influence ou de relations familiales plus efficaces, qui elles-mêmes résultent (dans les pays les moins développés, ou PMD) d'un accès plus précoce à l'instruction, aussi modeste puisse être celle-ci. L'élite des PMD et les classes dominantes de ces pays sont très largement le produit d'une opportunité historique qui leur a permis d'être instruits les premiers - beaucoup plus que d'une origine aristocratique ou de capacités intellectuelles exceptionnelles. Dans le cas du Soudan, quoi qu'il en soit, Niblock (1985) suggère que l'administration coloniale a consciemment favorisé les leaderships « historiques » (tribaux, confrériques, religieux...) en offrant à leurs fils (à travers l'éducation et les emplois gouvernementaux) l'occasion d'assurer leur pouvoir et leur domination.

5 Notre hypothèse est ici que les bénéfices de l'éducation peuvent être étendus aux contemporains (parents, amis, alliés, etc.) et, au fil du temps, aux enfants et petitsenfants à travers les comportements de " quête de rente " propres à ces élites (Krueger, 1974 ; Ashoff, 1989). Ainsi, le réseau des anciens élèves des premières promotions du Gordon Mémorial College (GMC) a permis à ceux-ci de se reproduire en tant que classe par le jeu de faveurs réciproques engageant l'attribution de postes lucratifs et/ou stratégiques sans tenir compte des coûts sociaux impliqués. La compétition pour l'emploi, qui aurait pu se développer en l'absence de tels monopoles, est ainsi constamment annulée et remplacée par le jeu des références et recommandations. Selon notre hypothèse, l'avantage économique qui accompagne le monopole du pouvoir d'État peut être incorporé à la rente résultant de façon générale des politiques interventionnistes de l'État. De ce point de vue, la rente associée au monopole du pouvoir d'État modifie les données du taux de profit personnel lié à un type donné d'éducation qui doit dès lors être envisagé en termes de bénéfice pour d'autres parents contemporains ou de générations à venir. Dans ce contexte, le modèle d'analyse en termes de "quête de rente" peut être utilisé pour tenter de comprendre les dysfonctionnements de l'économie et de la politique soudanaises.

6 La "quête de rente ", au sens qu'on lui donne ici, ne peut être réduite à un pur et simple égoïsme ou à un pur et simple opportunisme (Khalid, 1990). Il s'agit d'un comportement économique rationnel de la part d'un groupe de gens qui n'ont pas voulu laisser passer l'opportunité que leur offrait le hasard de l'histoire. De plus, l'ancrage social des membres de cette élite, le type et la qualité de l'éducation qu'ils ont reçue les empêchent de penser ou de se comporter autrement. La notion d'un Étatnation dont les intérêts prévaudraient sur ceux des individus ou des groupes leur est étrangère. D'où il résulte que les valeurs sociales dominantes contribuent à alimenter les comportements de «quête de rente » d'une élite «tribale ${ }^{2}$ restreinte qui voit ses 
intérêts dans la perpétuation des déséquilibres historiques existants. Le contrôle de la bureaucratie gouvernementale (choisir qui va occuper quel poste) apparait ici comme un enjeu trop stratégique pour être laissé aux considérations d'un intérêt national abstrait. Du coup, le développement (une meilleure utilisation des ressources disponibles) est bloqué par la perpétuation, plus ou moins consciente, des bases historiques de l'injustice.

Monopole du pouvoir d'état et avantage économique

7 Pour des raisons historiques - en particulier le fait que les autorités coloniales étaient peu désireuses de voir des néo-mahdistes être associés au nouvel État du Condominium (1898-1956) et préféraient, au moins au début, que le recrutement des agents du gouvernement s'opère parmi les éléments loyaux au nouveau régime ou parmi les ennemis avérés de l'État mahdiste défunt -, des écoles furent ouvertes d'abord à Khartoum et dans la province du Nord. Il est également important de rappeler que le Darfour ne fut intégré au Condominium qu'en 1916, et que c'est seulement en 1946 que le rattachement du Sud au Soudan - plutôt qu'au Kenya ou à l'Ouganda - fut définitivement acquis. Ces facteurs historiques, parmi d'autres, ont façonné le recrutement géographique des étudiants du Gordon Memorial College, comme le montre le tableau 1. On peut voir dans ce tableau que la majorité venait - et continuait à venir en 1989-90 - de Khartoum et des provinces du Nord et du Centre. La suprématie de ces régions peut être encore corroborée par les taux de concentration où Khartoum et la région du Nord occupent, en moyenne, environ $60 \%$ des places, et avec la province du centre jusqu'à plus de $85 \%$.

Tableau 1 : Origines des étudiants du Gordon Mémorial College (1929) et de l'Université de Khartoum $(1959,1989)$

\begin{tabular}{|c|c|c|c|c|c|c|c|}
\hline & 1929 & \multicolumn{2}{|c|}{$1959 / 1960$} & \multicolumn{2}{|c|}{$1989 / 1990$} & & \\
\hline & $\mathrm{Nb}$ & \begin{tabular}{l|l}
$\%$ & $n b$
\end{tabular} & $\%$ & $\mathrm{Nb}$ & $\%$ & & \\
\hline Khartoum & 218 & 42.7 & 399 & 33 & .3 & 1.006 & 47.2 \\
\hline Nord & 106 & 20.8 & 278 & 23 & .2 & 220 & 10.3 \\
\hline Centre & 134 & 26.3 & 295 & 24 & .6 & 625 & 29.3 \\
\hline Est & 11 & 2.2 & 66 & 5.5 & & 137 & 6.4 \\
\hline Kordofan & 41 & 8.0 & 90 & 7.5 & & 81 & 3.8 \\
\hline Darfour & - & - & 31 & 2, & & 56 & 2.6 \\
\hline Sud & - & - & 40 & 3. & & 1 & - \\
\hline Total & 510 & 100.0 & 1.199 & & 0.0 & 2.131 & 100.0 \\
\hline
\end{tabular}

Sources : Rapports annuels du GMC, statistiques de l'Education, comité d'admission 

monopole sur le pouvoir d'État, monopole identifié en termes de représentation régionale (participation) dans les gouvernements de la période postérieure à l'indépendance, d'accès aux postes dirigeants de la fonction publique, de l'armée, de la police, de la magistrature et de la diplomatie. La correspondance entre le monopole du pouvoir d'état et la concentration régionale de l'éducation est quasiment absolue, d'où l'on peut déduire que les autres facteurs sont probablement secondaires, ou du moins qu'un niveau minimum d'éducation est nécessaire pour avoir accès aux postes importants. Ces tableaux montrent, par exemple, que le Grand-Khartoum et la province du Nord ( $14 \%$ de la population totale) fournissent près de $80 \%$ des sous-secrétaires d'État et des chefs de département ministériels des autres services paragouvernementaux, $67 \%$ des hauts magistrats, $54 \%$ des officiers supérieurs et plus de $50 \%$ des ministres, officiers de police et ambassadeurs. La domination de ces deux régions sur le pouvoir d'état depuis l'indépendance, il y a 35 ans, peut ainsi être qualifiée de massive. Si l'on y inclut la région du Centre, l'indice de concentration du pouvoir d'Etal est, dans tous les domaines, au dessus de $70 \%$. En fait, la concentration parmi les officiers de l'armée au début des années 60 et dans les années 70 (tab. 3) est encore plus grande - plus de $70 \%$ pour les deux premières provinces, et plus de $80 \%$, en moyenne, si l'on intègre la troisième.

Encore la répartition des effectifs est-elle moins contrastée que celle des représentations relatives: si l'on considère la part des différentes régions dans l'accès aux positions dirigeantes de la fonction publique, les disparités sont encore plus accusées : la part de Khartoum est de 82 fois celle du Darfour pour ce qui concerne le nombre de sous-secrétaires d'État et de chefs de départements ministériels, 45 fois celle du Sud pour le nombre d'officiers supérieurs, et plus d'une fois et demie la part de l'ensemble des régions (sauf le Nord) pour le nombre des officiers de police. La part de la région du Nord, autre région sur représentée, correspond à 74 fois la part du Darfour, 7 fois celle de la région du Centre pour le nombre de sous-secrétaires d'État, 53 fois celle du Sud pour le nombre de hauts magistrats, et plus que l'ensemble des autres provinces (y compris Khartoum) pour le nombre d'ambassadeurs. Les faibles taux de représentation de certaines provinces vont jusqu'à l'exclusion pure et simple : le Sud ne compte ni sous-secrétaire d'État ni officier supérieur de police, et le Darfour n'a ni haut magistrat ni ambassadeur.

10 Pour mesurer à quel point est étroite la base du pouvoir (et de l'éducation), il n'est que de se reporter aux résultats du recensement de 1955-56 (Henin, 1963). Province de Khartoum : 4,9\% de la population totale ; Grand-Khartoum (246.000), 2,4 \% ; Omdurman (114.000), $1 \%$. Comme le montre le tableau, la ville d'Omdurman a fourni, entre 1954 et 1989, 34,5 \% des membres de cabinets ministériels, $25 \%$ des hauts fonctionnaires, 32,8 $\%$ et $18,9 \%$ des officiers supérieurs de la police et de l'armée, et $21,9 \%$ des hauts magistrats. A l'inverse, le Sud et le Darfour sont très faiblement représentés dans toutes ces catégories malgré leur poids dans la population globale (40.7\%).

11 La concentration du pouvoir d'État n'est pas seulement régionale mais aussi sociale et ethnique. Les tableaux 4 et 5 montrent la répartition sociale et ethnique des étudiants du GMC entre 1926 et 1944. Les dirigeants, dans tous les aspects de la vie soudanaise avant et après l'indépendance, sont issus de ce groupe. La majorité des étudiants, dans la période considérée, sont des Arabes (70 \%) et des Mawalid (22\%) et sont les fils de hauts fonctionnaires ou d'officiers de l'armée (entre $31 \%$ et $52 \%$ ) et de commerçants 
(21\%). L'administration du GMC était consciente de ce fait. Son rapport de 1930 note ainsi que "près de $70 \%$ des garçons sont d'origine arabe, et $50 \%$ sont fils de commerçants ou de fonctionnaires » (p. 15). En 1939-40, les fils de hauts fonctionnaires et d'officiers représentaient à eux seuls $53 \%$, pourcentage montant à $74 \%$ en y incluant les enfants de commerçants. Si l'on considère que, jusqu'en 1956, la population urbaine représentait 5,8\% de l'ensemble de la population du Soudan, on peut en conclure que la concentration de l'éducation chez les urbains, par opposition aux ruraux et aux nomades, était aussi extrêmement élevée.

Tableau 2 : Indicateurs de participation au pouvoir d'État selon la fonction et la région de provenance (1954-1989) *

\begin{tabular}{|c|c|c|c|c|c|c|c|c|c|c|c|c|c|c|c|c|c|c|c|}
\hline & \multicolumn{2}{|c|}{ Khartoum } & \multicolumn{2}{|c|}{ Nord } & \multicolumn{2}{|c|}{ Centre } & \multicolumn{2}{|c|}{ Est } & \multicolumn{2}{|c|}{ Kordofan } & \multicolumn{2}{|c|}{ Darfour } & \multicolumn{2}{|c|}{ Sud } & \multicolumn{2}{|c|}{ Autres ${ }^{* *}$} & \multirow{2}{*}{$\begin{array}{l}\text { Total } \\
\text { nb }\end{array}$} & \multicolumn{2}{|c|}{$\begin{array}{c}\text { Concentration } \\
\text { du pouvoir } \\
\text { d'Elat }\end{array}$} \\
\hline & $\mathrm{nb}$ & $\%$ & $\mathrm{nb}$ & $\%$ & $\mathrm{nb}$ & $\%$ & $\mathrm{nb}$ & $\%$ & nb & $\%$ & nb & $\%$ & $\mathrm{nb}$ & $\%$ & nb & $\%$ & & $\mathrm{nb}$ & $\%$ \\
\hline $\begin{array}{l}\text { 1. Ministres } \\
\text { 2- Sous-Sectétaires } \\
\text { et directeurs de }\end{array}$ & 126 & 45.3 & 29 & 10.4 & 40 & 14.3 & 15 & 3.4 & 27 & 9.7 & 9 & 3.2 & 30 & 10.8 & 2 & 0.7 & $\begin{array}{l}278 \\
\vdots \\
\end{array}$ & 55.7 & 70 \\
\hline départ. minist. & 74 & 50.0 & 42 & 28.4 & 20 & 13.5 & 2 & 1.4 & 9 & 6.1 & 1 & 0.7 & 0 & 0 & 0 & 0 & 148 & 78.4 & 91.9 \\
\hline 3. Officiers sup. armée & 122 & 48.8 & 38 & 15.2 & 33 & 13.2 & 13 & 5.2 & 26 & 10.4 & 8 & 3.2 & 8 & 3.2 & 2 & 0.8 & 250 & 64 & 27.2 \\
\hline 4. Otticiers sup. police & 33 & 29.7 & 30 & 27.0 & 21 & 18.9 & 15 & 13.5 & 8 & 7.2 & 5 & 4.5 & 0 & 0 & 0 & 0 & 111 & 56.7 & 25.6 \\
\hline 5- Juges Hte cour & 27 & 37.0 & 22 & 30.1 & 12 & 16.4 & 2 & 2.7 & 7 & 9.6 & 0 & 0 & 2 & 2.7 & 1 & 1.4 & 73 & 67.1 & 83.5 \\
\hline $\begin{array}{l}\text { 6- Ambassadeurs (1988) } \\
\text { 7. Hts tonctionnaires }\end{array}$ & 22 & 26.8 & 23 & 28.0 & 12 & 14.6 & 4 & 4.8 & 7 & 8.5 & 0 & 0 & 3 & 3.7 & 11 & 13.4 & 82 & 54.8 & 69.4 \\
\hline (1969-81) & 38 & 28.8 & 34 & 25.8 & 23 & 17.4 & 1 & 0.8 & 10 & 7.6 & 0 & 0 & 10 & 7.6 & 16 & 12.1 & 132 & 54.3 & 71.7 \\
\hline
\end{tabular}

Source : Study Research (1986-1990) \& Ibrahim (1985) pour la colonne 7 (Sud).

* Données pour la période 1954-1989, sauf indications contraires et col. 7. Echantillons de 52 \% pour les hauts-fonctionnaires, de $90 \%$ pour les ambassadeurs (en 1988) et de $70 \%$ pour les magistrats \& officiers.

** Y compris les personnes nées hors du Soudan et celles dont le lieu de naissance n'est pas précisé. 
Tableau 3 : Distribution régionale des officiers de l'armée soudanaise (1962 - 1972)

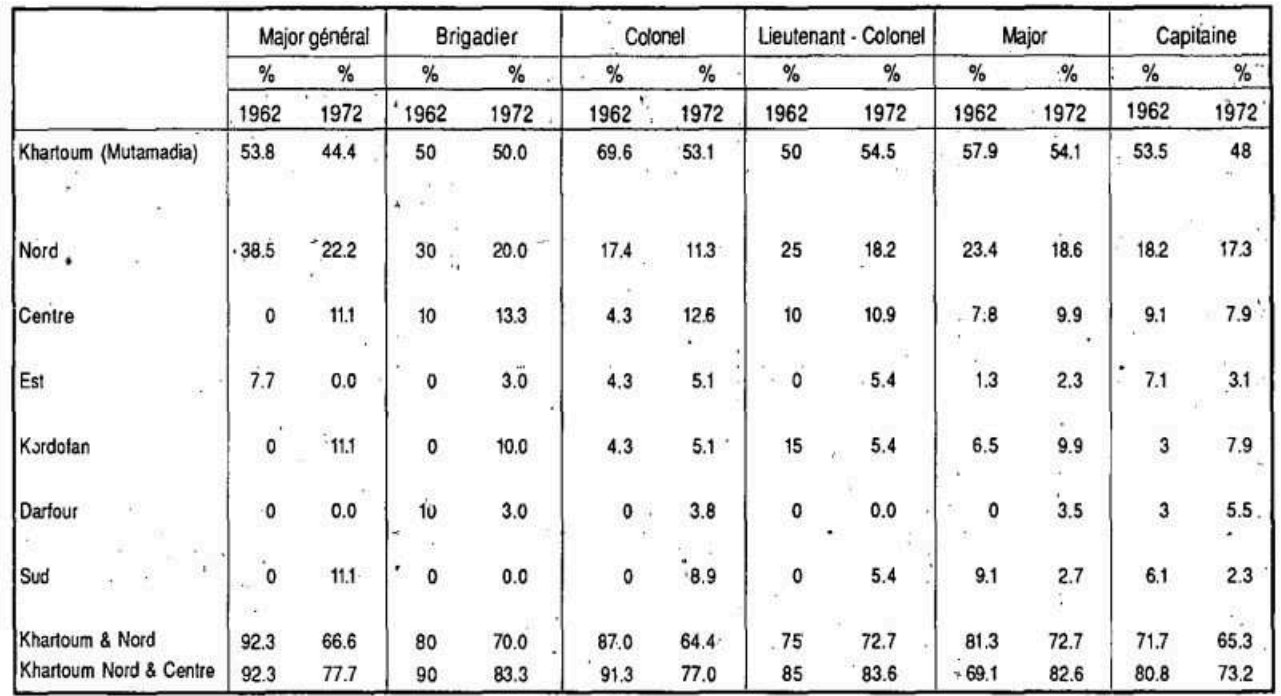

Source : Study Research (1986-1990). Echantillon 1962; 419 personnes. Echantillon 1972 : 472 personnes.

Tableau 4 : Origine ethnique des étudiants du Cordon Memorial College (1926-1944)

\begin{tabular}{|c|c|c|c|c|c|c|}
\hline & \multicolumn{2}{|c|}{$1926 \cdot 1930$} & \multicolumn{2}{|c|}{$1937-1940$} & \multicolumn{2}{|c|}{$1941-1944$} \\
\hline & nb & $\%$ & nb & $\%$ & nb & $\%$ \\
\hline d'origine arabe & 1.536 & 70.5 & 1.055 & 66 & 1.475 & 70.5 \\
\hline d'orig. africaine 1 & 71 & 3.3 & 41 & 2.6 & 30 & 1.4 \\
\hline Mawalid ${ }^{2}$ & 442 & 20.3 & 387 & 24.2 & 456 & 21.8 \\
\hline Barabra $^{3}$ & 123 & 5.6 & 102 & 6.4 & 106 & 5.1 \\
\hline Autres étrangers ${ }^{4}$ & 8 & 0.3 & 14 & 0.9 & 27 & 1.3 \\
\hline Total & 2.180 & 100 & 1.099 & 100 & 2.049 & 100 \\
\hline
\end{tabular}

Source : GMC, Rapport annuel 1941, p. 21, \& 1925-1930.

1) Egalement désignés dans certains rapports comme Arabes du sud.

2) Sont ainsi désignés les Soudanais d'ascendance étrangère (égyptienne, turque, syrienne, grecque...)

Dans certains rapports, ils sont désignés comme mustawtinin ou muwalladin.

3) On trouve cette désignation, et non celle de Nubiens, dans tous les rapports.

4) II s'agit d'étudiants éthiopiens, yéménites et somaliens. 


\begin{tabular}{|l|rr|rr|}
\hline & \multicolumn{2}{|c|}{$1926-1930$} & \multicolumn{2}{c|}{$1939-1940$} \\
& $\mathrm{nb}$ & $\%$ & \multicolumn{1}{c|}{ nb } & $\%$ \\
\hline Fonctionnaires & 608 & 27.8 & 1.467 & 48.9 \\
Officiers & 74 & 3.4 & 86 & 2.9 \\
Commerçants & 466 & 21.4 & 619 & 20.6 \\
Chels de tribu & 88 & 4.0 & 67 & 2.2 \\
Artisans & 165 & 7.6 & 338 & 11.3 \\
Agriculteurs & 788 & 35.7 & 426 & 14.2 \\
Total & 2.113 & 100.0 & 3.003 & 100.0 \\
\hline
\end{tabular}

Source : GMC, Annual Reports 1926-1944. Nuzzar et Umad.

Les statistiques de l'éducation pour 1959-60 montrent que la situation n'a pas changé, quinze ans après, pour ce qui concerne les étudiants de l'université de Khartoum : 43,7 $\%$ sont fils de hauts fonctionnaires ou d'employés du secteur public, 31,7 \% fils de commerçants ou d'hommes d'affaires, $16,8 \%$ fils d'agriculteurs et 7,8 \% fils d'ouvriers. Ainsi, aucun des biais (géographique, ethnique, social et urbain) n'a été corrigé au fil des années. Sur la base de ces chiffres, on peut dégager un certain nombre de caractéristiques prédominantes concernant l'élite au pouvoir dans le Soudan d'après l'indépendance. Les membres de cette élite sont généralement "nordistes", Arabes, résidant pour la plupart dans les zones urbaines, ou à défaut, sur les rives nilotiques du Nord-Soudan, et appartenant aux strates supérieures de la société soudanaise, en substance la bureaucratie gouvernementale et la classe des commerçants. Ces données sont de nature à appuyer les propositions de Shaddad (1988), el-Bathani (1988) et Ibrahim (1985) quant à la nature du groupé dominant (la classe capitaliste araboislamique).

Dans les États en voie de développement, l'État assume des rôles qui concernent tous les aspects de l'existence : permis, licences, accès à la terre - agricole ou constructible subventionnée par le gouvernement, au crédit subventionné, accès aux marchandises et aux services contrôlés par le gouvernement, etc., dépendant étroitement de la bureaucratie. En fait, dans les pays en voie de développement, le gouvernement est en situation de promouvoir, ou au contraire de faire obstacle à toute initiative au sein du secteur privé ; c'est pourquoi contrôler la bureaucratie d'État signifie être assis sur une mine d'or. Comme le montre Shaddad (1988), l'accès à « la bureaucratie d'État, qui est la véritable source de la richesse et du contrôle sur le pouvoir, est la principale condition pour une accumulation primitive « parasitaire » de ressources ». Qu'il en soit ainsi, la classe commerçante dont les fils représentent eh moyenne 25 \% des diplômés du GMC ne sont pas les seuls à en être conscients : c'est cet état d'esprit qui constitue la 
«tribu des diplômés » elle-même. Comme l'écrit Ibrahim (1985), ses membres étaient, dès 1942 , tout à fait conscients de "l'importance de la bureaucratie comme source de revenu, de statut et de pouvoir ».

Nous nous proposons d'examiner dans ce qui suit quelques exemples de bénéfices accumulés par le biais de contrôles sur la bureaucratie gouvernementale. Des emplois relativement lucratifs sont ainsi la chasse gardée du groupe dominant, les "diplômés » et leurs alliés, la "classe commerçante », pour leur permettre de se reproduire et de conserver les moyens de cette domination. Ces emplois vont des postes de commandement dans l'armée, la banque, les sociétés du secteur public ou paraétatique permettant de dégager d'importantes marges bénéficiaires, jusqu'au secteur privé, banques, compagnies étrangères et organisations régionales et internationales. Les réseaux d'anciens élèves sont ainsi à l'œuvre et déterminés à conserver un avantage économique qui n'est jamais acquis sur la base du seul "mérite ». Il est sans doute difficile de calculer le taux de rapport de l'éducation intermédiaire et supérieure (au GMC), au cours de cette période, mais il ne fait pas de doute qu'il était très élevé,

Le tableau 6 montre les taux de concentration régionale pour un certain nombre d'emplois relativement bien rémunérés, en particulier ceux offerts par les banques, l'administration de la Gezira, les organisations régionales ayant leur siège à Khartoum, la Banque arabo-africaine pour le développement et les emplois des diplômés à l'étranger. Encore une fois, il y a une correspondance directe entre la concentration historique de l'accès à l'éducation dans la zone riveraine du Nil du Nord-Soudan et le contrôle des emplois les plus lucratifs. On peut sans doute s'attendre à retrouver les mêmes taux pour ce qui concerne les postes gouvernementaux et le recrutement étatique au Soudan et à l'étranger.

Tableau 6 : Concentration régionale des cadres et employés (1989)

\begin{tabular}{|c|c|c|c|c|c|c|c|c|c|c|}
\hline & \multirow{2}{*}{\multicolumn{2}{|c|}{$\begin{array}{l}\text { Secteur } \\
\text { bancaire }\end{array}$}} & \multirow{2}{*}{\multicolumn{2}{|c|}{$\begin{array}{l}\text { Périmètre } \\
\text { agric. Gezira }\end{array}$}} & \multicolumn{6}{|c|}{ BADEA * } \\
\hline & & & & & \multicolumn{2}{|c|}{ Cadres sup. } & \multicolumn{2}{|c|}{ Cadres moy. } & \multicolumn{2}{|c|}{ Employés } \\
\hline & nb. & $\%$ & nb. & $\%$ & nb. & $\%$ & nb. & $\%$ & nb. & $\%$ \\
\hline Khartoum & 28 & 32.2 & 59 & 18.2 & 13 & 56.4 & 14 & 43.8 & 18 & 66.7 \\
\hline Nord & 18 & 20.7 & 52 & 16.0 & 4 & 17.4 & 17 & 53.1 & 6 & 25.9 \\
\hline Centre & 21 & 24.1 & 175 & 53.8 & 2 & 8.7 & 0 & 0.0 & 1 & 3.7 \\
\hline Est & 6 & 6.9 & 10 & 3.1 & 1 & 4.3 & 0 & 0.0 & 0 & 0.0 \\
\hline Kordolan & 5 & 5.7 & 12 & 3.7 & 2 & 8.6 & 0 & 0.0 & 0 & 0.0 \\
\hline Darfour & 2 & 2.3 & 3 & 1.0 & 1 & 4.3 & 1 & 3.1 & 2 & 7.4 \\
\hline Sud & 6 & 6.9 & 0 & 0.0 & 0 & 0.0 & 0 & 0.0 & 0 & 0.0 \\
\hline Autres & & 1.1 & - & - & - & & - & & - & - \\
\hline Total & 87 & 100.0 & 325 & 100.0 & 23 & 100.0 & 32 & 100.0 & 27 & 100.0 \\
\hline
\end{tabular}

Source : Study research (1986-1990). Banque afro-arabe de développement. 

dans le personnel d'une organisation régionale telle que la Banque arabo-africaine pour le développement, connue pour payer les salaires les plus élevés de la région.

Un autre exemple d'avantage économique est la distribution des fermes mécanisées. Un accès au crédit distribué par la Banque agricole du Soudan, et/ou aux fonds accordés par la Banque mondiale, fait partie - entre autres avantages - de ces "apanages" attribués par le gouvernement. Il existe des exemples de candidatures émanant de gens totalement non qualifiés et qui aboutissent à l'attribution, à de hauts fonctionnaires gouvernementaux à la retraite, à d'anciens officiers ou à des marchands urbains, de fermes mécanisées à Habila dans le sud du Kordofan. Le tableau 7 montre la distribution des unités productives de la région d'agriculture mécanisée de Habila par antécédents professionnels.

Tableau 7 : Appartenance professionnelle des titulaires d'exploitations dans le périmètre agricole de Habila (1974)

Source : Ibrahim H. E., Agricultural Development Policy, Ethnicity and Socio-Political Changes in the Nuba Mountains, Ph. D. Thesis (Connecticut) USA, 1988, p. 128.

Deux observations doivent être ajoutées. En premier lieu, une grande partie de ces acquéreurs de fermes sont par ailleurs officiers de l'armée en retraite. Ceux-ci, et d'autres, louent le plus souvent à des commerçants, en l'occurrence des commerçants urbains d'al-Obeid, qui appartiennent par leur origine ethnique au Nord-Soudan nilotique. Même sans tenir compte de ces locations, la concentration des fermes atteint plus de dix exploitations par investisseur. En second lieu, le phénomène des propriétaires absentéistes est dominant. Ibrahim (1988) rapporte qu'en 1982 cet absentéisme concernait 57,6 \% des projets soutenus par la Banque mondiale et $24 \%$ des projets financés par la Banque agricole du Soudan. Ceci tend, bien sûr, à justifier les récriminations des populations locales quant à l'inéquité du système de distribution de ces projets d'agriculture mécanisée. Celte-ci est centralisée par la Mechanized Farming Corporation (MFC) dont le siège est à Khartoum. Notons que le commissaire en charge de la province du Sud-Kordofan a été privé de son siège au Conseil des directeurs de la MFC, en même temps que de celui qu'il occupait au Comité pour la répartition des fermes mécanisées, et que ceci n'est sans doute pas sans raison. Dans le même ordre d'idées, l'Union des cultivateurs des Monts Nouba, ayant pris conscience de ces malversations, a envoyé en 1974 une lettre de protestation à la MFC, où l'on peut lire «qu'il est devenu évident pour tout le monde que la distribution des fermes mécanisées dans la région de Habila s'est opérée en toute inégalité et en toute injustice. Cette discrimination est fondamentalement dirigée contre les populations locales des Monts Nouba.» (Ibrahim, 1988, p. 112-115) Ces protestations, et d'autres similaires, sont restées quoi qu'il en soit lettre morte, pour des raisons sans doute plus fortes que les exigences de la construction nationale.

Le tableau 8 montre comment des individus modestement éduqués mais occupant des postes à responsabilité recrutent de préférence du personnel faiblement qualifié plutôt que des candidats plus compétents. On remarque dans ce tableau que la majorité des hauts dirigeants des unités considérées (banque. administration de la Gezira, directeurs de la fonction publique) ont de faibles qualifications universitaires - scolarité secondaire ou de niveau inférieur. Ceci ne signifie pas que la détention de titres universitaires soit une garantie de gestion efficace, mais ce point méritait d'être relevé.

Égypte/Monde arabe, 15-16 | 1993 
Des administrateurs ou des dirigeants pourvus de faibles qualifications ne peuvent recruter ni admettre un personnel plus qualifié qu'eux. Un autre indice de cette gestion à courte vue serait, si elle était disponible, une cartographie des investissements publics. Le volume des ressources affectées au sous-secteur irrigué moderne, et la négligence symétrique qui entoure le sous-secteur d'agriculture pluviale alors que celui-ci constitue le principal fondement de l'économie et concerne la majorité de la population, en constitue une illustration significative.

Tableau 8 : Niveau d'études des cadres de différentes institutions

\begin{tabular}{|c|c|c|c|c|c|c|c|c|}
\hline & \multicolumn{2}{|c|}{$\begin{array}{c}\text { Déparlemenls } \\
\text { ministériels } \\
1965 / 66\end{array}$} & \multicolumn{2}{|c|}{$\begin{array}{c}\text { Périmètre de } \\
\text { la Gezira } \\
1989\end{array}$} & \multicolumn{2}{|c|}{$\begin{array}{c}\text { Banque de } \\
\text { Khartoum } \\
1990\end{array}$} & \multicolumn{2}{|c|}{$\begin{array}{c}\text { Banque de } \\
\text { P'Unité } \\
1989\end{array}$} \\
\hline & & $\%$ & $n b$ & $\%$ & $n$ & $\%$ & $\overline{n b}$ & $\%$ \\
\hline Secondaire ler cycle & 27 & 47.4 & 36 & 10.8 & 29 & 34.5 & 12 & 8.4 \\
\hline Secondaire $2 \theta$ cycle & 15 & 26.3 & 210 & 63.7 & 31 & 36.9 & 120 & 84.5 \\
\hline Universitaire & 15 & 26.3 & 79 & 24.0 & 24 & 28.6 & 10 & 7.1 \\
\hline Total & 57 & 100 & 325 & 100 & 84 & 100 & 142 & 100 \\
\hline
\end{tabular}

Source : Study research (1986-1990) ; Civil Service Committee (1967), Final Report.

Implications en termes de mauvaise gestion de l'économie

Une utilisation rien moins qu'optimale des ressources humaines entraîne des coûts significatifs pour l'économie. Les notations qui précèdent montrent que tel a bien été le cas au Soudan. Le recrutement aux postes de direction du service public et du secteur privé est, dans une large mesure, déterminé par des considérations indépendantes de la qualification (relations familiales, tribales, confessionnelles). Mais cela est encore plus vrai pour le recrutement et/ou la promotion aux postes à haute responsabilité dont l'attribution fait rarement l'objet de compétition. De telles pratiques engendrent une instabilité permanente au sommet de l'administration. A chaque changement de gouvernement (de régime), les hauts fonctionnaires se préparent à être remplacés; II y a là une situation contraire au pur et simple bon sens, dans la mesure où le recrutement sur des bases compétitives assure une plus grande stabilité, autorise à:attendre des comptes et favorise l'efficacité dans l'utilisation des ressources disponibles.

21 La sous-utilisation des ressources humaines a des effets plus durables qu'une mauvaise gestion d'autres ressources, tels que l'eau ou les équipements. Tandis que les coûts de la seconde peuvent être évalués en termes de perte de productivité, la première (c'est-àdire le recrutement de dirigeants incompétents) signifie la réduction des possibilités mêmes de production (l'efficacité) dans le temps, qui s'étend aux autres ressources commandées par les activités concernées - ce qui signifie l'enfermement de l'économie et l'inhibition de ses opportunités de développement et de progrès.

La " tribu des diplômés ", avec son étroite base socio-ethnique et ses comportements de "recherche de rente", concentre dans ses mains la distribution de ressources et l'opportunité même de créer des ressources aux dépens de l'économie dans son ensemble. Le modeste niveau de qualification de la première génération de cadres - la "Soudana Generation» - a agi comme un frein au recrutement des individus les mieux 
qualifiés et/ou a contribué à la frustration de ceux qui étaient malgré tout recrutés en diminuant leurs chances de promotion. Ce dernier aspect s'est révélé particulièrement dévastateur en démoralisant nombre de fonctionnaires, incités à exercer leurs activités à un niveau largement inférieur, à leurs compétences. Les nouvelles recrues doivent en effet se montrer «flexibles» et accepter de brader certains de leurs principes et/ou leur intégrité, simplement pour survivre.

L'attribution de postes à responsabilité à un personnel sous-qualifié et l'imposition de bas critères professionnels aux nouveaux recrutés pour les inciter à se conformer à un modèle peu exigeant du service public ont fait que l'économie a fonctionné en deçà de ses possibilités productives plutôt qu'à leurs limites - pour ne pas parler ici de l'extension de ces possibilités. De façon générale, on peut faire l'hypothèse que les chances de développement continueront à être gaspillées tant que de larges segments de la société se sentiront privés de la juste part qui leur revient dans le partage du "gâteau national ». Un tel sentiment de frustration peut se traduire par des sabotages et déboucher sur des affrontements violents dès lors qu'il est devenu évident que la répartition géographique des investissements publics est biaisée et ne peut se justifier ni en termes de taux de profit ni sur les bases de l'intérêt national.

Même s'il est vrai qu'une distribution équitable des postes (représentation dans l'appareil gouvernemental) entre les régions et les groupes ethniques pourraient ne pas être suffisante en elle-même pour améliorer les chances de développement, ceci doit être tenté dans la logique d'un passage à un système plus transparent et compétitif. Il ne serait peut-être pas mal avisé de commencer par des mesures de discrimination positives en faveur des régions et des groupes marginalisés avant que d'instaurer un système d'allocation des ressources purement compétitif.

\section{BIBLIOGRAPHIE}

Ashoff G., « Rent-Seeking ; A New Concept in the Economic Theory of Politics and the Debate on Development Theory », Economies, vol. 40,1989, pp. 7-41.

Bhagwati J.M., « Education Class Structure and Income Inequality », World Development (1973) in Bhagwati J. M. (1985), Wealth and Poverty, Basil Blackwell, 1985.

Civil Service Committee, Final Report, vol. 1 (limited circulation — in arabic), 1967.

El-Bathani A., « Nation Building between Democracy and Dictatorship », paper presented to Erkawit 11th Conference (in arabic), nov. 1988.

Governor General of Sudan, Annual Reports (1908-1941).

Gordon Memorial College, Annual Reports (1901-1946).

Henin R.A, « Economic Development and Internal Migration in Sudan », Sudan Notes and Records. 1963; pp. 100-119.

Ibrahim A. A., Regional Inequality and Underdevelopment in Western Sudan, Ph. D thesis Universily of Sussex,1985. 
Ibrahim H. E., Agricultural Development Policy, Ethnicity and Socio-Political Changes in the Nuba Mountains, Ph.D thesis, Connecticut, 1988.

Khalid M., The Government they Deserve : The Role of the Elite in Sudan's Political Evolution, Kegan Paul, 1990.

Krueger A., « The Political Economy of Rent-Seeking Society », AER, vol. 64, 1974, pp. 291-303.

Niblock T., Class and Society in Sudan, London, 1985.

Shaddad M.Z. ", Al-Sudan wa Azmat al-Hukm [Sudan and the Governing crisis], Dar al-Nasag, 1988.

\section{NOTES}

1. Le présent article s'appuie largement sur la première partie d'une étude réalisée par l'auteur sous le titre «Education et mauvaise gestion de l'économie et de la société soudanaises, 1954-1989 », Document de travail du DSRC, 88,1990.

2. SALIH Mahjûb M., rédacteur en chef du quotidien al-Ayyâm, est l'inventeur du terme «tribu des diplômés» qu'il décrit comme étant à la fois peu nombreux mais très puissants. AI-Ayyâm, 9/2/88.

INDEX

Mots-clés : Soudan

\section{AUTEUR}

SIDDIG UMBADDA

Université de Khartoum 